\title{
Thermal Insulation of Smart Energy Coating for Energy Production
}

\author{
Irina Pospelova \\ Department of Engineering Services and Life-Support \\ Systems \\ Irkutsk National Research Technical University \\ Irkutsk, Russia \\ pospel@istu.edu \\ Denis Kornilov \\ Department of Engineering Services and Life-Support \\ Systems \\ Irkutsk National Research Technical University \\ Irkutsk, Russia
}

\author{
Angelina Bondarenko \\ Department of Engineering Services and Life-Support \\ Systems \\ Irkutsk National Research Technical University \\ Irkutsk, Russia
}

\author{
Maria Pospelova \\ Department of Engineering Services and Life-Support \\ Systems \\ Irkutsk National Research Technical University \\ Irkutsk, Russia
}

\begin{abstract}
Developed SmartEnergyCoating can be used as a thermal insulation coating. This is able to compensate and equalize thermal loads of structural elements of constructions. The effect is achieved due to the heat-compensating elements with the phase-transition material. In addition to thermal protection properties, the coating is capable of generating energy independently. A thermoelectric heat pump operates by the Peltier effect.
\end{abstract}

Keywords-Smart Networks, Smart Coating, Alternative Energy

\section{INTRODUCTION}

The ecological situation in the world is characterized by a rapid increase in energy consumption, including renewable energy. The development of technologies leads to an increase in the burden on the environment. This causes the need to search for new technologies to restore and maintain the ecological balance. The SmartEnergyCoating is environmental friendly. It allows reducing chemical and thermal emissions into the atmosphere, while increasing the amount of electricity produced autonomously. allows to provide independent power production in any field conditions.

In remote areas from the centralization, there are difficulties of production and delivery of energy in sufficient quantities. This is due to the bed quality of the pipelines, and sometimes the absence of the need to make capital constructions in temporary work or remoteness from centralization. In these conditions, it is necessary to invent devices that can generate electricity independently without using expensive fuel or fossil energy sources.

The authors developed a coating that can be applied to various surfaces, and capable of generating energy based on the thermoelectric effect. This coating is capable to generate electricity independently without a fossil fuel energy sources and even the sun, wind and other sources. Thermally conductive inclusions of the inside layer with a phase transition material ensure the stable operation of the Peltier element. In addition the generation of electricity, it ensures a stable temperature regime of work for any structures due to inclusions with a phase transition.

The advantages of the developed system are following. It is the only suitable way of obtaining energy in a small space. There is absence of moving parts; it makes the system highly reliable. It is not necessary to change the refrigerant (freon, because it is not in the design). There is no sensitivity to vibrations (important for transport). There is the possibility of a smooth and precise regulation of the temperature regime and ecological compatibility. Thermoelectric devices do not contain toxic refrigerants. It can have an arbitrary orientation in space and a gravity field. It has a small inertia, switches from cooling mode to heating mode easily.

\section{MODE OF STABILIZATION OF THE TEMPERATURE FIELD}

Phase transitions allow maintaining a stable temperature regime in the thickness and on the surface of structural elements and providing a stable temperature regime for Peltier elements.

Heat-storage accumulation is based on the ability of substances to store energy with heating and cooling. In this case the amount of accumulated energy depends on the temperature of the heat-accumulating material, and its specific heat. Accumulation of thermal energy, based on the use of a reversible process of the melting-solidification phase transition, is an effective way of keeping heat. In this case, the phase-transfer material is used as heat-storage material. Per unit volume in phase transitions a greater amount of heat is stored than without a phase change. The charging and discharging process can be carried out in a close limit temperature range. It is very important when it is necessary to operate heat accumulators in conditions of small temperature limits. [1]. 
A class of accumulators with phase transitions can be divided for the production of heat and cold with a wide range of operating temperatures $\mathrm{T}$. It is low-temperature processes $\left(20^{\circ} \mathrm{C} \leq \mathrm{T}<200^{\circ} \mathrm{C}\right)$, medium temperature $\left(200^{\circ} \mathrm{C} \leq \mathrm{T}<500^{\circ}\right.$ $\mathrm{C})$, high-temperature $\left(500^{\circ} \mathrm{C}<\mathrm{T}\right)$.

The low temperature installations are used most widely, because it uses in human life support systems, they have environmental friendly methods of energy production and optimization of energy consumption. The use of mediumtemperature installations is associated with power plants mainly and heat recovery systems. High-temperature installations are known in the application in metallurgy and power engineering.

To model and estimate an accumulate system with a phase transition, it is necessary to know the features of the behavior of systems in which a phase transition from the solid phase to the liquid phase and back is carried out [2,3].

Depending on the behavior of the solid phase transition to the liquid phase or back, the process realizes with absorption or release of heat. This heat is called the latent heat of the phase transition. It is expended on the destruction of the crystal lattice during melting and it is released during crystallization. The specific heat (per $\mathrm{kg}$ of weight) of the phase transition for a given substance is constant as:

$$
\mathrm{Q}=\mathrm{Q}_{\mathrm{ph}} / \mathrm{m}
$$
mass.

where $\mathrm{Q}_{\mathrm{ph}}$ - the latent heat of phase transition; $\mathrm{m}$ - the

If the process is carried out at a constant pressure, then $\mathrm{Q}_{\mathrm{ph}}$ is constant and equal to the enthalpy of the phase transition $\Delta \mathrm{H}_{\mathrm{ph}}$.

In addition, in inorganic substances, the temperature $\mathrm{T}_{\mathrm{ph}}$ at which the phase transition occurs is also constant. $T_{p h}$ is called the phase transition temperature. The quantities $\Delta \mathrm{H}_{\mathrm{ph}}$ and $\mathrm{T}_{\mathrm{ph}}$ for various substances can be found in chemical reference books or reference books of physical quantities.

Based on the heat balance for the phase interface, the Stefan condition can be derived [4]:

$\rho_{\text {тв }} \mathrm{Q}_{\mathrm{p}} \partial \mathrm{s}_{\mathrm{n}} / \partial \tau=\lambda_{\mathrm{g}} \partial \mathrm{T} /\left.\partial \mathrm{n}\right|_{\mathrm{n} \rightarrow+0}-\lambda_{\text {тв }} \partial \mathrm{T} /\left.\partial \mathrm{n}\right|_{\mathrm{n} \rightarrow-0}$ (2)

where $s_{n}$ - the moving of demarcation line of phases to a direction of a normal; $\tau$ - time.

Relation (2) allows calculating the displacement of the boundary of the phase transition over the temperature fields in the liquid and solid phases. At the same time, the movement of the interfacial surface affects to the temperature fields.

According to authors, the problem of modeling of possible ways for installing of phase transition inclusions in "Smart Cover" was set. In addition, the thermal protection of the coating must have good thermal conductivity at the locations of the Peltier elements.

In addition to generating electric power, Smart Cover keeps the heat received and further compensates for the temperature difference to maintain the stability of the set temperature, and thereby prolongs the time of independent generation of electricity. Special nanostructural elements allow absorbing excess heat and, if necessary, transfer it to the elements that produce electrical energy. Thus, structural elements, such as valves, test equipment and other elements are in the permissible temperature regime and receive additional energy.

The heat flux along the layer is distributed depending on the presence or absence of internal sources of heat release or absorption, described by a simple Fourier equation or by adding to it the heat of the internal source. In the absence of internal sources of heat release or absorption, the differential heat equation (Fourier equation) is derived:

$$
\frac{\partial t}{\partial \tau}=\frac{\lambda}{c \rho} \frac{\partial^{2} t}{\partial x^{2}}
$$

where $\frac{\lambda}{c \rho}=a, \mathrm{~m}^{2} / \mathrm{h}-$ the coefficient of thermal diffusivity, $\mathrm{c}$ - isobaric specific heat, $\rho$ - density.

In the most general form, with unsteady propagation of heat along all three axes of coordinates, the differential heat equation becomes a three-dimensional form:

$$
\frac{\partial t}{\partial \tau}=a\left[\frac{\partial^{2} t}{\partial x^{2}}+\frac{\partial^{2} t}{\partial y^{2}}+\frac{\partial^{2} t}{\partial z^{2}}\right]=a \Delta^{2} t
$$

where $\Delta^{2}=\frac{\partial^{2}}{\partial x^{2}}+\frac{\partial^{2}}{\partial y^{2}}+\frac{\partial^{2}}{\partial z^{2}}$ is the Laplace operator.

In an inhomogeneous material medium, the differential equation of a three-dimensional temperature field will have the form:

$$
\frac{\partial}{\partial x}\left(\lambda_{x} \frac{\partial t}{\partial x}\right)+\frac{\partial}{\partial y}\left(\lambda_{y} \frac{\partial t}{\partial y}\right)+\frac{\partial}{\partial z}\left(\lambda_{z} \frac{\partial t}{\partial z}\right)=0
$$

where $\lambda$ is a continuous function of $x$ and $y$.

Spatial temperature fields for practical purposes in most cases are sufficient to study in individual projections, which can be represented by two-dimensional temperature fields.

In the presence of a source with a phase transition material in which the temperature change front passes along equation (1), we can write the equations for determining isothermal lines as:

$$
\frac{\partial t}{\partial z}=a_{1} \frac{\partial^{2} t}{\partial x^{2}}+a_{2} \sqrt{t}
$$

where $a_{1}, \mathrm{~m}^{2} / \mathrm{h}$ - coefficient of thermal diffusivity of nonphase-transition layers, $a_{2}$ is the thermal diffusivity parameter for a layer with a phase transition [5].

As a result of a change in the volume of the liquid-solid phase system, the position of the outer boundaries is changed gradually. With sufficient duration of the process, it affects to the configuration of the volume form of the phase-transition material.

In many problems of the Stefan type [6-8], free convection in the liquid phase cannot be neglected. The heat exchange in this case multiplies many times. The melting process can proceed under conditions of a fixed solid phase or in a free state. Most substances in the solid phase sinks under the action of gravity, the exception being water. Accordingly, convection is considered with a fixed or loose solid phase. In such problems, heat transfer in the liquid phase is described by a system of equations of convective heat transfer.

In this case, we envisage dimensions for phase-change inclusions no more than permissible for the exclusion of convection. 


\section{MODELING}

Depending on the external boundary conditions and operating conditions, we can calculate the location of the thermal inclusions for the optimum performance of the structure.

Figure 1 shows the simulation of the total heat flux and the location of the phase-transition inclusions.

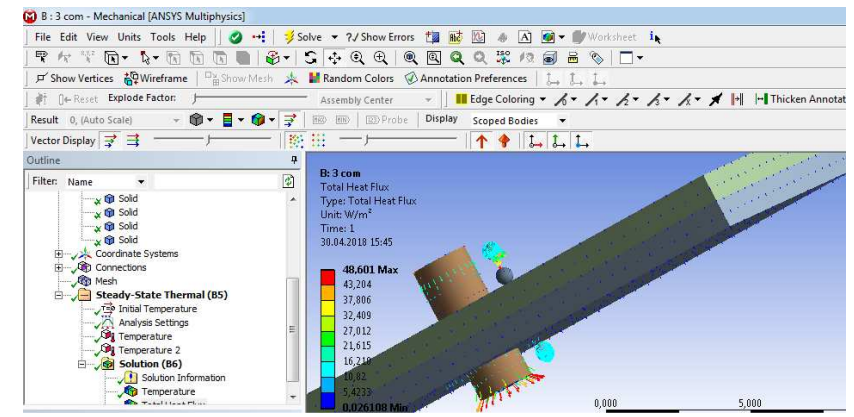

Fig. 1. The location of the phase-transfer layer is shown in blue. Brown color - parts of heat-conducting inserts for Peltier elements.

Figure 2 shows a smooth temperature change in the thickness of the "Smart Cover". Despite the set temperature difference of 20 and $-20^{\circ} \mathrm{C}$, the coating smoothly maintains the temperature difference sufficiently and retains the specified difference. At the same time, in places with Peltier elements, the temperature difference remains at the required level.

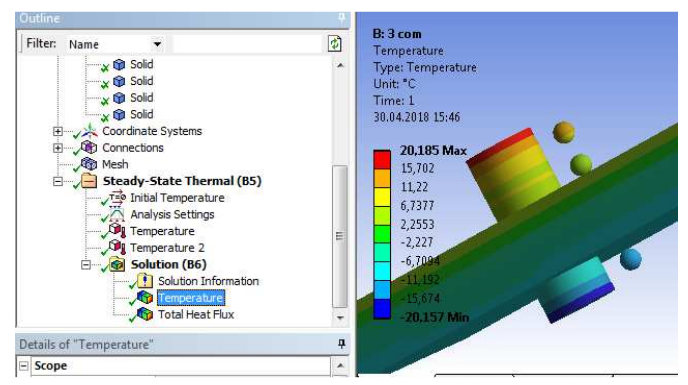

Fig. 2. Distribution of temperature lines inside the coating.

Figure 3 shows the value of the temperature head in the thickness of the coating and in the places of heat-conducting elements. Elements with a specified accuracy conduct a temperature level within the required limits and with a minimum temperature difference. This makes it possible to obtain a given temperature difference for generating electricity.

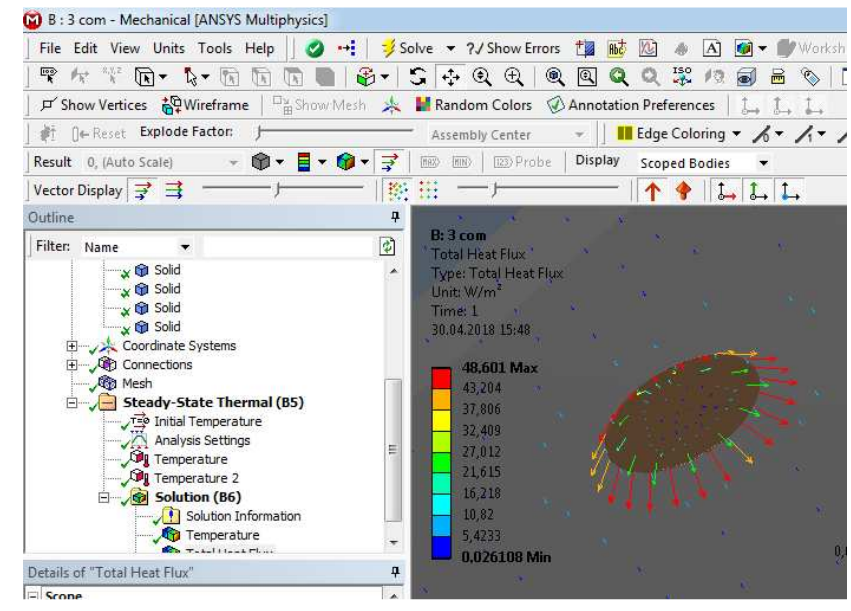

Fig. 3. Location of the Peltier element for generating electricity

Within the framework of this simulation, the operating conditions of the equipment, similar to the Siberian climatic conditions, were set.

Thus, thermal inclusions with a phase-transition material for boundary conditions corresponding to Siberian conditions, with an average minimum ambient temperature of $-22^{\circ} \mathrm{C}$ according to the simulation data, amount to 4-5 $\mu \mathrm{m}$ with variable pitch, calculated depending on the required coating thickness and internal temperature. The total mass and propagation of the phase-transition material in the final sample can be calculated more accurately with the help of electronic crystal and thermography in the future.

After determining the location of the isothermal lines on the surface and in the thickness of the material, we can talk about generating electricity $[9,10]$.

The energy production modes were modeled for different compositions. One of the options was a design with an outer layer with good absorption coefficients and minimal reflection of the outer layers of the structure. The properties of the constituent elements of the layer are similar to those of graphene analogs. This allows you to receive heat energy as much as possible.

In the simulation, the heat-absorbing outer layer was taken into account as a layer of absorption and heat. As a thermal insulator in which the material with a phase transition is located, a conventional polyurethane foam layer, or any other material with good thermal insulation properties and price criteria, was adopted. As a composite material with a phase transition, a heat-accumulating material with inclusions based on paraffin in polyethylene capsules was adopted. The size of the capsules varied as a result of the experiments.

\section{CONCLUSIONS}

Based on the results of thermal modelling and several fullscale experiments, we can say that the developed thermal insulation layer allows one to distribute the temperature field through the thermal conductive inclusions so well the value of the temperature difference arises directly in places with Peltier elements. 
The remaining areas are reliably protected from excessive heat flux. Such coating can be used in building structures, in pipeline systems of hot water supply and heating. At the same time, the drop in temperature for thermal power generation will be insignificant, and the output voltage is sufficient for the operation of equipment, lighting, and other energy users.

\section{References}

[1] N. Rossikhin, "Features of calculation of heat accumulators on phase transitions with an intermediate coolant," Engineering Journal: Science and Innovation. Electronic scientific and technical publication. Vol. 5(17), 2013. DOI: 10.18698/2308-6033-2013-5-727

[2] L. Rubinshtein, The Stefan problem, Riga: Zvayzgne, 1970.

[3] E. Kartashov, Analytical methods in the theory of the thermal conductivity of solids, Moscow: "High School", 1985.

[4] E. Eckert, R. Drake, Theory of heat and mass transfer, Moscow: Gosenergoizdat, 1961.

[5] I. Pospelova, "Determination of Optimal Heat-Storage Thickness of Layer for "Smart Wall" by Methods of Nonlinear Heat Conduction Equations for Phase-transition Materials," IOP Conference Series: Materials Science and Engineering. International Conference on Construction, Architecture and Technosphere Safety (ICCATS 2017)
21-22 September 2017, Chelyabinsk, Russian Federation. Retrieved from: http://iopscience.iop.org/article/10.1088/1757899X/262/1/012031.

[6] L Martinson, Y Malov, Differential equations of mathematical physics Moscow: Moscow State Technical University of Bauman, 2002.

[7] E. Kosova, V. Lebedev, "Model of a structural first-order phase transition close to the second," J. of Fundamental Research, Vol. 2 (7), pp. 70-71, 2006.

[8] V. Alekseev, A. Carabin, "New type of thermal accumulators for cooling radioelectronic equipment for space vehicles," Electronic Journal Proceedings of the MAI, Vol. 49, 2010. Retrieved from: https://mai.ru/upload/iblock/2b8/novyy-tip-teplovykh-akkumulyatorovdlya-okhlazhdeniya-radioelektronnoy-apparatury-kosmicheskikhapparatov.pdf

[9] V. Khvesyuk, D. Ostanko, A. Scriabin, P. Tsygankov, R. Chelmodyev, A. Chirkov, "Limiting efficiency of thermoelectric heat conversion in high-temperature power plants," Science \& Education of Bauman. MSTU, Vol. 3, pp 81-105, 2016.

[10] V. Kulbachinskiy, V. Kytin, V. Blank, S .Buga, M. Popov, "Thermoelectric properties of nanocomposites of bismuth telluride with fullerenes," Physics and Technology of Semiconductors, Vol. 45(9), pp. 1241-1245, 2011. 